\title{
TSP-1 Mimetic ABT-510
}

National Cancer Institute

\section{Source}

National Cancer Institute. TSP-1 Mimetic ABT-510. NCI Thesaurus. Code C37447.

A synthetic peptide that mimics the anti-angiogenic activity of the endogenous protein thrombospondin-1 (TSP-1). ABT-510 inhibits the actions of several pro-angiogenic growth factors important to tumor neovascularization; these pro-angiog enic growth factors include vascular endothelial growth factor (VEGF), basic fibroblast growth factor (bFGF)), hepatocyte growth factor (HGF), and interleukin 8 (IL-8). (NCI04) 